\title{
Handheld Laparoscopic Forceps Manipulator Using Multi-slider Linkage Mechanisms
}

\author{
Hiromasa Yamashita ${ }^{1}$, Nobuhiko Hata ${ }^{1}$, Makoto Hashizume ${ }^{2}$, and \\ Takeyoshi Dohi ${ }^{1}$
}

1 Graduate School of Information Science and Technology, The University of Tokyo,

7-3-1 Hongo Bunkyo-ku, Tokyo, 133-8656, Japan

\{hiromasa, noby, dohi\}@atre.t.u-tokyo.ac.jp

http://www.atre.t.u-tokyo.ac.jp

2 Graduate School of Medical Sciences, Kyushu University,

3-1-1 Maidashi Higashi-ku, Fukuoka-shi, Fukuoka, 812-8582, Japan

mhashi@dem.med.kyushu-u.ac.jp

http://www . camit.org/hospital

\begin{abstract}
This paper proposes a new handheld laparoscopic forceps manipulator using 2-DOFs bending mechanism by multi-slider linkage mechanisms and 1-DOF wire-driven grasping mechanism. Careful design of the linkage channels enables unique and independent bending procedure from -90 to 90 degrees on the horizontal and vertical plane to secure large workspace. The manipulator consists of multi-DOFs end-effector, linear-drive unit, dial-type interface and computer-based control unit. In mechanical performance analyses, 2-DOFs bending mechanism enabled high accuracy of less $1.0 \mathrm{~mm}$ manipulation and high bending power of up to $0.85 \mathrm{kgf}$. In vivo experiments, this manipulator performed laparoscopic surgical tasks, such as raising the liver and the stomach, and suturing the stomach surface tissue in $22.3 \pm 5.4$ seconds per suture. Furthermore, we operated cholecystectomy with an animal within 45 minutes. In conclusion we were sure of a usefulness of a new handheld laparoscopic forceps manipulator for speedy and dexterous laparoscopic surgery.
\end{abstract}

\section{Introduction}

Laparoscopic surgery enables the incision on abdominal wall smaller, thus making invasion to patients minimal. For the sake of this advantage laparoscopic surgery is taken in almost all surgery, such as abdominal surgery, chest surgery, obstetrics and gynecology. After securing space below abdominal wall, surgeons insert special surgical instruments such as forceps and electric cauteries through trocars, and operate instruments under laparoscopic control. However, surgical approaches and manipulations are restricted due to low degree-of-freedom (DOF) instruments . This inflexibility, combined with limited laparoscopic view of the operative field, causes surgeons' mental and physical stress.

To overcome the issues on limited maneuverability in the abdominal cavity, several robotized devices have been proposed to add additional degree-of-motion at the tip of the forceps [1]-5]. The da Vinci ${ }^{\text {TM }}$ Surgical System by Intuitive 


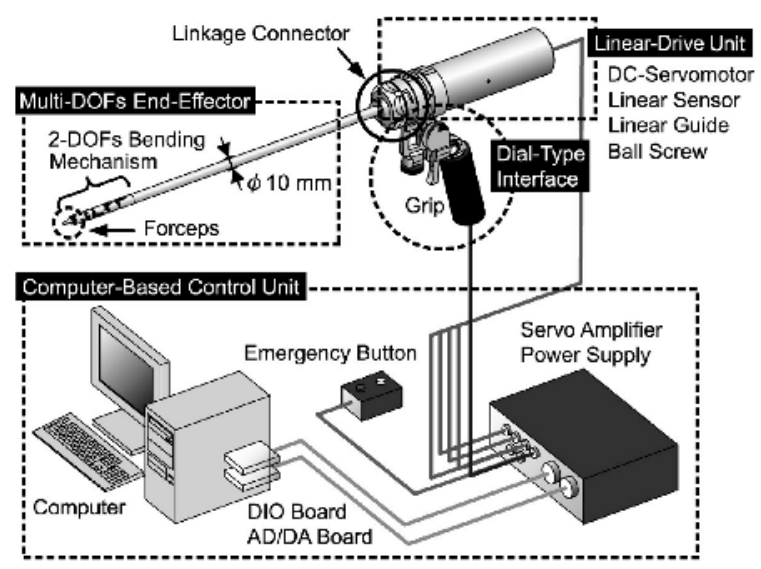

Fig. 1. System configuration of the handheld laparoscopic forceps manipulator.

Surgical Inc. enabled minimal invasive tele-surgery with miniature wire-driven multi-DOFs instruments having near-wrist manipulation [1]. The Mechatronic Arthroscope by Dario et al. enabled unique steering mechanism with two actuating cables, whose external diameter was $4 \mathrm{~mm}$, steering range was 110 degrees and the mean error in the accuracy was 1.1 degrees [2]. These approaches proposed so far utilized wire-driven mechanism to transfer actuation power from actuators outside the abdominal cavity to actuated parts in the tip of the forceps. While this choice wire-driven mechanism is relatively straightforward approach, the issues on wearing and extension of wire has been unsolved.

Our proposal in this paper is to use multi-slider linkage mechanisms instead of wire-driven mechanisms to achieve 2-DOFs motion at the tip of the laparoscopic forceps. The linkage-driven approach is known to have high stiffness, durability and accuracy in manipulation [6] 7]. The engineering contribution of this paper is slider-linkage drive to enable robotized motion in the forceps, especially the careful placement design of two slider-linkages while achieving no interference of motion each other. This paper is clinically significant since the highly accurate and stiff 2-DOFs motion of forceps tip possible by newly developed multi-slider linkage mechanisms enable speedy and dexterous laparoscopic tasks such as raising, suturing and ligation. This paper reports 1) newly developed multi-slider linkage mechanism based on our previous preliminary work [8, 2) development of handheld laparoscopic forceps manipulator using this linkage mechanism, 3) in vivo experiments and animal cholecystectomy study for evaluation.

\section{Methods}

\subsection{System Configuration}

The system configuration of the handheld laparoscopic forceps manipulator consisted of mainly four parts (Fig. 11). First part was the multi-DOFs end-effector 
with 2-DOFs bending mechanism and 1-DOF grasping mechanism. Second part was the linear-drive unit consisted of three sets of brushless DC-servomotors (FAULHABER GROUP MINIMOTOR SA, 1628024 B), linear sensors detecting linkage displacements (ALPS ELECTRIC CO., LTD., RDC1014A09), linearguides (THK Co., Ltd., RSR3WNUU+36L+) and ball-screws (NSK Ltd., M3 × 0.5). Third part was the dial-type handheld interface with three spindle operated potentiometers (Meggitt Electronic Components Ltd., TYPE 51 SERIES) consisted two dials for the horizontal and vertical bending, a trigger for grasping and a button for straightening the bending mechanisms in getting through a trocar. Fourth part is the computer-based control unit consisted a computer (CPU: Intel Pentium 4 2.00 GHz., RAM: 512 MB, OS: RedHat Linux 7.3) and three servo amplifiers (FAULHABER GROUP MINIMOTOR SA, BLD 3502) calculating displacements of sliding two linkages and one stainless-steel wire by inputted target angles from the dial-type interface.

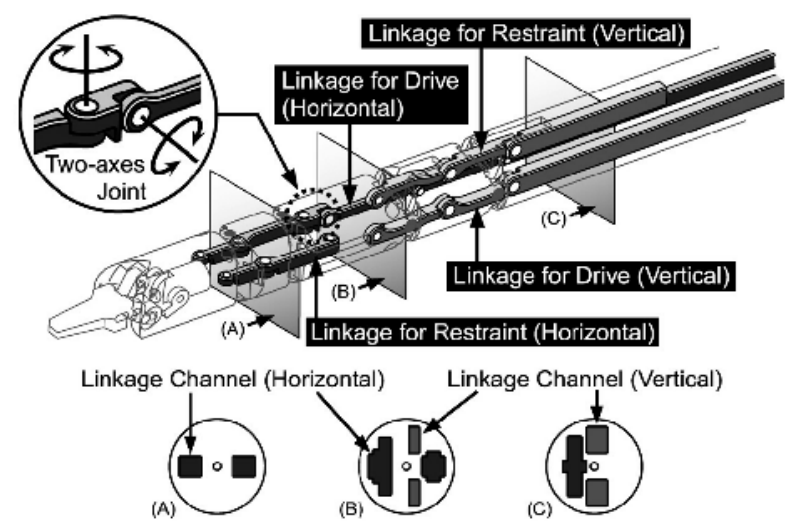

Fig. 2. Perspective view of the 2-DOFs bending mechanism that consists of five frames, two sets of linkages for drive and restraint. Each linkage slides through inner channel shown in sections (A), (B) and (C). Two-axes Joint connects the linkage for drive in horizontal bending mechanism and three links toward the actuator, enabling two-axes rotation responding to the vertical bending angle.

\subsection{2-DOFs Bending Mechanism}

2-DOFs bending mechanism for the multi-DOFs end-effector was constituted by multi-slider linkage mechanisms. These mechanisms consisted of five outer cylindrical frames, four joints and two sets of linkage mechanisms, one was for the horizontal bending and the other was for the vertical bending (Fig. 2). Each linkage mechanism, furthermore, consisted of two linkages, one was for drive outer frames and the other was for restraint enabling unique driving procedure between \pm 90 degrees and independent 2-DOFs bending manipulation. Constituent materials of 2-DOFs bending mechanism were all stainless-steel, outer frames and joint-pins were SUS304 and inner linkages were SUS316 (Fig. 3). 


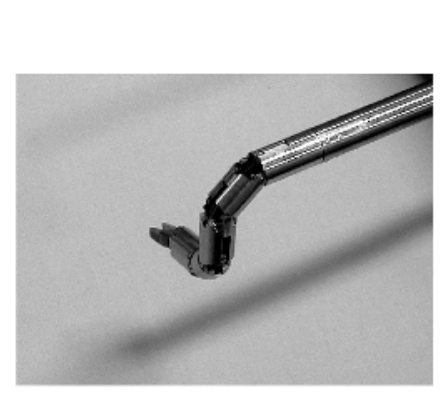

(A)

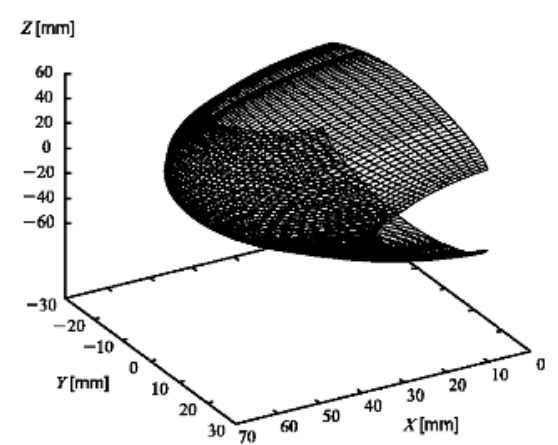

(B)

Fig. 3. (A) View of the 2-DOFs bending mechanism bending 90 degrees in horizontal plane and -90 degrees in vertical plane. (B) Workspace of the end-effector with 2-DOFs bending manipulation covering almost half surface of an oval sphere, whose origin of the coordinates is at an actuator-side of vertical bending mechanism.

\section{Results}

\subsection{Mechanical Performance}

We examined repeatability and response characteristic of the 2-DOFs bending mechanism using digital video camera (Sony Corporation, DCR-PC110), and generated power at the tip of an end-effector.

Measurements of repeatability were done unloaded with five trials each bending mechanism, examining the actual bending angles against target angles inputted from the computer with five times (Table 1).

Table 1. Results of repeatability measurement in each bending mechanism.

\begin{tabular}{lcc}
\hline Measurement item & Horizontal bending Vertical bending \\
\hline Repeatability(mean of SD) & $\pm 0.87^{\circ}$ & $\pm 0.91^{\circ}$ \\
Hysteresis error & less $9.0^{\circ}$ & less $5.5^{\circ}$ \\
Error over the theoretical value & less $13.3^{\circ}$ & less $10.1^{\circ}$ \\
\hline
\end{tabular}

As response characteristics, we examined actual bending angle against input target angle from dial-type interface to evaluate delay times of 2-DOFs bending mechanisms. Fig. 4 shows results of response characteristics of each bending mechanism between input bending speed and delay time.

3-DOFs generated powers at the tip of an end-effector and calculated torque at the rotational axes are shown in Table 2. Powers of all DOFs and directions fulfilled $0.40 \mathrm{kgf}$ of a requested specification and achieved up to sufficient values of $0.85 \mathrm{kgf}$ for laparoscopic surgery. 


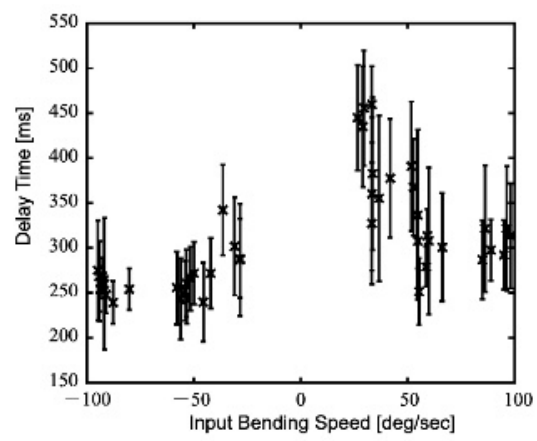

(A)

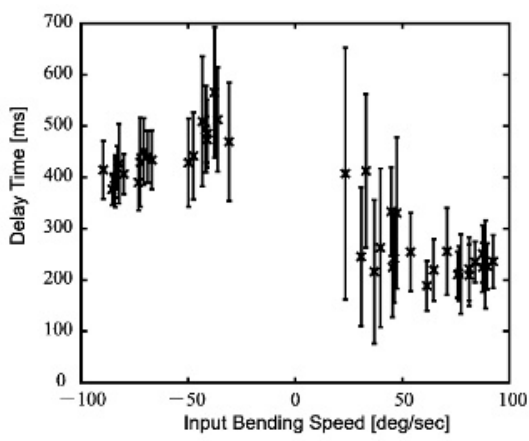

(B)

Fig. 4. Response characteristics of (A) the tip-side horizontal bending mechanism and (B) the base-side vertical bending mechanism.

Table 2. Generated power and torque at the end-effector.

\begin{tabular}{llcc}
\hline DOF & Direction & Power $[\mathrm{kgf}]$ & Torque $[\mathrm{mNm}]$ \\
\hline Horizontal & $0^{\circ} \rightarrow 90^{\circ}$ & 0.70 & 118.6 \\
Horizontal & $0^{\circ} \rightarrow-90^{\circ}$ & 0.85 & 144.1 \\
Vertical & $0^{\circ} \rightarrow 90^{\circ}$ & 0.40 & 165.7 \\
Vertical & $0^{\circ} \rightarrow-90^{\circ}$ & 0.50 & 207.1 \\
Grasp & Closing & 0.85 & 60.8 \\
\hline
\end{tabular}

\subsection{In Vivo Experiments}

We did usability analyses of the manipulator in near-clinical setting. We had surgeons approach to the liver and the stomach on an animal (swine, $42.5 \mathrm{~kg}$, male) under pneumoperitoneum and do some laparoscopic surgical tasks shown in Fig. 5] Especially in suturing task, we confirmed smooth procedure of one suture in $22.3 \pm 5.4$ seconds on an average of nine times.

\subsection{Cholecystectomy}

As an usability analysis of the manipulator for more practical surgical works, we operated cholecystectomy with an animal (swine, $43.0 \mathrm{~kg}$, male) under pneumoperitoneum (Fig. 6). It took 44 minutes and 30 seconds from laparoscope insertion into abdominal cavity to completion of gallbladder ablation.

\section{Discussion}

\subsection{Mechanical Performance}

We confirmed that larger workspace of \pm 90 degrees in 2-DOFs bending mechanism with high repeatability of less \pm 1.0 degree at the end-effector. Repeata- 


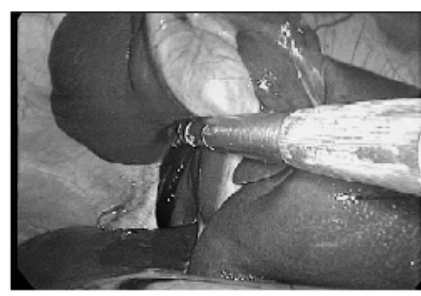

(A)

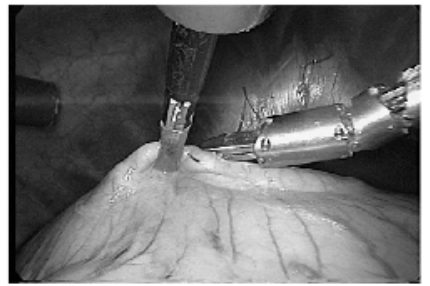

(C)

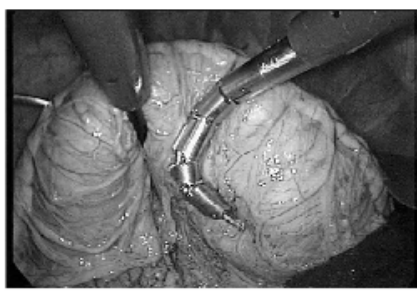

(B)

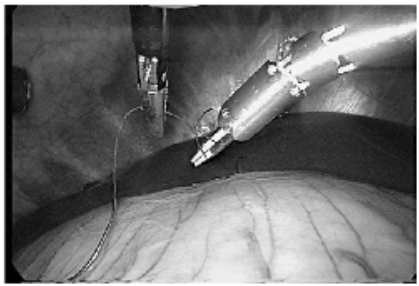

(D)

Fig. 5. (A) Circumventing, holding and raising the liver without flexure and deformation of the elements of bending mechanisms. (B) 2-DOF bending angles fit to the stomach curved surface and raising the whole stomach by the safe approach. (C) Stitching the surface tissue before suturing task from appropriate aproach directions with tip side bending motion. (D) Winding a thread around the end-effector, grasping and pulling the thread with tip side DOF manipulation to complete one suture task.

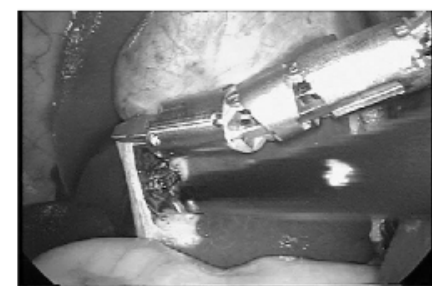

(A)

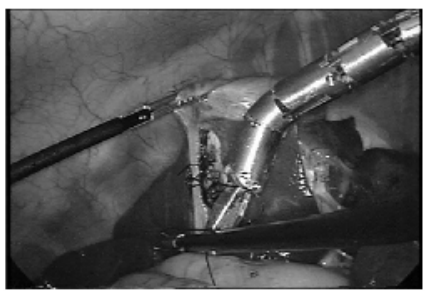

(C)

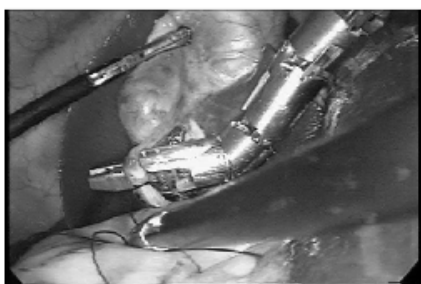

(B)

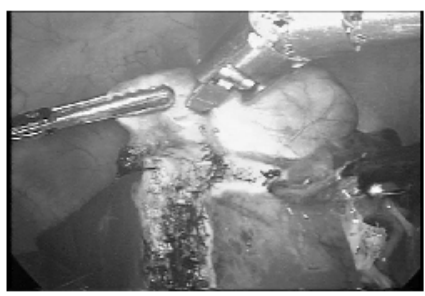

(D)

Fig. 6. (A) Grasping and raising the cystic duct for ablation with the electric cautery. (B) Passing the manipulator's tip part through ablated cystic duct with horizontal swing motion to grasp and pull a thread. (C) Ligation of the cystic duct. One ligation took $26.9 \pm 7.11$ seconds on an average of seven times. (D) Ablation of the gallbladder with the manipulator, a conventional forceps and an electric cautery. 
bility of \pm 0.87 degrees on the horizontal bending equaled $\pm 0.47 \mathrm{~mm}$ and \pm 0.91 degrees on the vertical bending equaled $\pm 0.89 \mathrm{~mm}$ at the end-effector, which demonstrated that plays of multi-slider linkage mechanisms were quite little. Response characteristics varied according to bending DOFs, directions and input bending speed from dial-type interface. Differences affected by the first and second factors were caused by the number of links in 2-DOFs bending mechanism and length of linkage path in cylindrical frames. About third factor, blushless DC-servomotors' operating limit influenced linkage speeds, particularly in case input bending speed was very slow. On the other hand delay time in each DOF often broke $200 \mathrm{~ms}$, which is said to be delay in human perception 9. Doctors evaluated, however, that they didn't feel noticeable delay in operation of the manipulator. Generated powers of all DOFs and directions fulfilled requested specifications, however, also varied according to bending DOFs and directions. Bending powers from 0 to 90 degrees were $80 \%$ of powers from 0 to \pm 90 degrees, which indicated that the driving efficiency of pushing linkages was $80 \%$ of one of pulling linkages. Considering torques at rotational joints, calculated by generated powers, driving efficiency of the tip-side horizontal bending was $70 \%$ of one of the base-side vertical bending, which caused the number of links in the linkage for drive.

\subsection{In Vivo Experiments}

From the results of in vivo experiments we were sure of the usability of a handheld laparoscopic forceps manipulator through some surgical tasks. Bending powers up to $0.85 \mathrm{kgf}$ were sufficient for raising and holding the liver and the stomach from a reverse side. Grasping power up to $0.85 \mathrm{kgf}$ was stronger than conventional forceps to grasp a thin thread in suturing tasks, however, knurled surface, wide and short shape of tooth were not suitable for grasping large tissues and curved needle. As the 2-DOFs bending manipulation, the base-side DOF aided in flexible and safe approaches to target affected area in the abdominal cavity, and tip-side DOF advantaged dexterous work after approaches. However, we confirmed that additional DOFs such as rotation and swing are necessary to the end-effector for more effective, quick and precise operation.

\subsection{Cholecystectomy}

We operated cholecystectomy in near-clinical setting with an animal within 45 minutes, which was faster than normal operation time of one hour on the average. In the recently reported result of animal study with a surgical robot [5], the average cholecystectomy time for the six animal cases was 47 minutes, we found our handheld laparoscopic forceps manipulator to be useful for more practical clinical application.

\section{Conclusion}

We were sure of the usefulness of newly developed handheld laparoscopic forceps manipulator with large workspace, high repeatability of less $1.0 \mathrm{~mm}$ and sufficient power of up to $0.85 \mathrm{kgf}$ at the end-effector. Especially in vivo experiments 
we operated a cholecystectomy within 45 minutes, which was speedy compared with conventional surgery, and high mechanical performance of the manipulator enables advanced laparoscopic surgery.

Acknowledgment. We wish to thank Mr. Iimura and Mr. Nakazawa of THK Co., LTD for cooperation in development of the linear-drive unit and the linkage connector. A part of this work is supported by Research for the Future Program "Development of Surgical Robot", administered by Japan Society for the Promotion of Science and Industrial Technology Research Grant Program in '03 from New Energy and Industrial Technology Development Organization (NEDO) of Japan.

\section{References}

1. Abbou, C., C., Hoznek, A., Salomon, L., Olsson, L., E., Lobontiu, A., Saint, F., Cicco, A., Antiphon, P., Chopin, D.: Laparoscopic radical prostatectomy with a remote controlled robot. JOURNAL OF UROLOGY. 165 (2001) 1964-1966

2. Dario, P., Carrozza, M., C., Marcacci, M., Attanasio, S., D'., Magnami, B., Tonet, O., Megali, G.: A Novel Mechatronic Tool for Computer-Assisted Arthroscopy. IEEE Trans. Inform. Technol. Biomed. 4(1) (2000) 15-28

3. Nakamura, R., Oura, T., Kobayashi, E., Sakuma, I., Dohi, T., Yahagi, N., Tsuji, T., Hashimoto, D., Shimada, M., Hashizume, M.: Multi-DOF Forceps Manipulator System for Laparoscopic Surgery - Mechanism miniaturized \& Evaluation of New Interface -. Proc. of 4th International Conference on Medical Image Computing and Computer-Assisted Intervention. (2001) 606-613

4. Ikuta, K., Sasaki, K., Yamamoto, K., Shimada, T.: Remote Microsurgery System for Deep and Narrow Space - Development of New Surgical Procedure and Microrobotic Tool. Proc. of 5th International Conference on Medical Image Computing and Computer-Assisted Intervention. (2002) 163-172

5. Butner, S., E., Ghodoussi, M.: Transforming a Surgical Robot for Human Telesurgery. IEEE Trans. Robot. Automat. 19(5) (2003) 818-824

6. Peirs, J., Reynaerts, D., Van Brussel, H.: A miniature manipulator for integration in a self-propelling endoscope. Sensors and Actuators A. (2001) 343-349

7. Kobayashi, Y., Chiyoda, S., Watabe, K., Okada, M., Nakamura, Y.: Small Occupancy Robotic Mechanisms for Endoscopic Surgery. Proc. of 5th International Conference on Medical Image Computing and Computer-Assisted Intervention. (2002) 75-82

8. Yamashita, H., Kim, D., Hata, N., Dohi, T.: Multi-Slider Linkage Mechanism for Endoscopic Forceps Manipulator. Proc. of the 2003 IEEE/RSJ International Conference on Intelligent Robots and Systems. 3 (2003) 2577-2582

9. Bate, L., Cook, C.: The Feasibility of Force Control Over the Internet. Proc. of the 2001 Australian Conference on Robotics and Automation. (2001) 146-151 